\title{
Introduction to the Minitrack on IT Governance and its Mechanisms
}

\author{
Wim Van Grembergen \\ University of Antwerp - Antwerp Management School \\ Wim.vangrembergen@uantwerpen.be
}

To be innovative and competitive in today's global digital economy, organizations have little choice but to invest in information and communication technologies (IT). However, without the proper organizational capabilities and skills to put these digital assets to effective use, organizations are at significant risk of wasting their investments and missing key opportunities for growth and competitiveness.

Against this context, the "IT governance and its mechanisms" research mini-track focuses on the design and implementation of organizational capabilities and e-leadership skills required to be successful in digital innovation and transformation journeys.

Since 2001, we are chairing this "IT Governance and its mechanisms" research track at HICSS, in order to advance research and practice in this area. Already with a tradition of more than 15 years, the research track focuses on the organizational challenges of governing and managing the digital assets and enterprise. In this knowledge area, the emphasis is on how organizations enable both business and IT people to execute their responsibilities in support of business/IT alignment and the creation of business value from IT-enabled investments. [1]

This research track is part of our activities within the IT Alignment and Governance Research Institute (ITAG) of the University of Antwerp and Antwerp Management School. Our aim is to develop state-ofthe-art concepts, models and theories that help organizations to generate more value out of IT enabled investments while managing its business risks. ITAG wants to be an international point of reference that

\author{
Steven De Haes \\ University of Antwerp - Antwerp Management School \\ steven.dehaes@uantwerpen.be
}

creates, distributes and applies innovative research that is grounded in science and rooted in practice.

The 2018 edition of the HICSS mini-track "IT governance and its mechanisms" is reporting on new and innovative insights that are on the research agenda of the knowledge area. We have received 17 valuable papers reporting on full research or research in progress, of which 7 were accepted after the reviewing process (acceptance rate $+/-41 \%$ ). Of course, we would like to acknowledge all the contributing authors to our research track and look forward to receiving new research submissions next year.

In this year's contributions, a lot of emphasis is put on the role, design and operating of some IT governance organizational structures and processes as antecedent towards alignment and firm performance. Specific focus areas include how key themes such as Devops and Big Data are to be positioned within the IT governance debate. Also, different viewpoints are analyzed regarding how IT governance implementation lead towards firm performance, considering elements such as culture, CIO advise, alignment in complex settings and shared understanding between business and IT. Finally, progression is proposed to bring more theory in the further development of IT governance constructs, with specific attention towards Viable Systems Theory.

Below, you will find an overview of the titles and authors of the 2018 edition of our research track, including the abstracts.

We look forward to discussing these topics with you further at the conference, and receiving your contributions of new innovative work in the future.

Using the Viable System Model to Study IT Governance Tim Huygh, Steven De Haes Dynamics: Evidence from a Single Case Study

An Empirical Assessment of Shared Understanding in IT Anant Joshi, Tim Huygh, Steven De Haes, Wim Governance Implementation

Motivating CIO Advice Networking to Improve Firm Jeffrey J. Pittaway, Hamed Qahri-Saremi, Ali Performance Reza Montazemi

The Complementarity of Corporate IT Alignment and Business Magno Queiroz, Tim Coltman, Paul Tallon, Unit IT Alignment: An Analysis of Their Joint Effects on Rajeev Sharma, Peter Reynolds Business Unit Performance 
Information Governance in the Big Data Era: Aligning Patrick Mikalef, John Krogstie, Rogier van de Organizational Capabilities $\quad$ Wetering, Ilias Pappas, Michail Giannakos

IT Governance Mechanisms for DevOps Teams - How Anna Wiedemann

Incumbent Companies Achieve Competitive Advantages

How Does the Organizational Culture of Collaborative Networks Parisa Aasi, Lazar Rusu, Dorothy Leidner, Erik Influence IT Governance Performance in a Large Organization? Perjons, Martha Corrales Estrada

Using the Viable System Model to Study IT Governance Dynamics: Evidence from a Single Case Study

This paper presents a single descriptive case study in which the viable system model (VSM) is used as a theoretical lens to model an organization's contemporary IT governance system. The case presented herein was selected specifically for being a digitized company of which we knew that a lot of effort was put recently in their IT governance system. We find that the case company's IT governance system maps well to the structure and underlying logic of the VSM. This paper contributes to the literature by providing an empirical justification exemplar on the applicability of systemic thinking in general, and the VSM in specific, for modelling enterprise governance and management of IT.

\section{An Empirical Assessment of Shared} Understanding in IT Governance Implementation

Drawing on literature about social alignment and shared understanding between different internal stakeholders, this study sets out to analyze the association between stakeholder functional role and the perceived level of IT governance implementation. Specifically, this paper takes a COBIT 5 perspective, by first analyzing perception differences at the level of the implementation of the seven COBIT 5 enablers, followed by an analysis at the level of the five COBIT 5 process domains. The results indicate that shared understanding about the IT governance implementation level between different organizational stakeholders can be improved, especially between (1) IT and (2) audit, risk, and compliance (ARC) stakeholders.

\section{Motivating CIO Advice Networking to Improve Firm Performance}

Prior research has examined factors that motivate executives in general to seek advice from external peers. The literature is silent however regarding what IT governance mechanisms motivate CIOs to mindfully seek advice in their external advice networks. Our research shows that simply applying the advice of the prior literature in the CIO and IT governance context can be counterproductive. We analyze data from a survey of 99 municipal corporations to show that an effective configuration of IT governance mechanisms fits and changes over time with the firm's financial performance and state of IT enactment. The lessons are instructive for practitioners, while also highlighting the importance of attending to context in IT governance research.

The Complementarity of Corporate IT Alignment and Business Unit IT Alignment: An Analysis of Their Joint Effects on Business Unit Performance

Alignment between IT and business strategy is a perennial challenge for IT executives, in part due to the evolving nature of organizational structure. In multi-business organizations (MBOs), a pressing issue for IT executives is how to improve the performance of each strategic business unit (SBU). In this paper we examine how IT alignment in MBOs affects SBU performance. We distinguish between IT alignment at the corporate and SBU levels and propose that these two types of IT alignment are complementary and exert joint effects on SBU performance. Two hypotheses related to these joint effects are developed and tested using data collected from an international survey of IT executives. Our findings indicate that complementarities between corporate IT alignment and SBU IT alignment enhance SBU performance. The primary contribution of this paper is explaining how different types of IT alignment in MBOs - individually and jointly affect SBU performance.

IT Governance Mechanisms for DevOps
Teams - How Incumbent Companies Achieve
Competitive Advantages
More and more organizations are deciding to
move from traditional, plan-driven software
development to agile approaches in order to stay
competitive. Therefore, the IT functions have been
deciding to implement cross-functional DevOps
teams. To enable collaboration within DevOps teams,
incumbent companies have to implement
mechanisms to govern dynamic and agile
environments. The present research investigates
which IT governance mechanisms are helpful for the
implementation of DevOps teams. For this purpose,
we conducted a qualitative research study and
interviewed team members in six companies that
have already implemented DevOps-oriented teams.


We describe which IT governance mechanisms - in the form of structure, processes, and relational mechanisms - are important for DevOps teams to achieve competitive advantages. Our findings show that agile roles and responsibilities, hybrid or decentralized organizational structures, as well as communications and knowledge-sharing models are conducive to the government of a DevOps team.

Information Governance in the Big Data Era: Aligning Organizational Capabilities

With big data growing rapidly in importance, academics and practitioners have been considering the means through which they can incorporate the shifts these technologies bring into their competitive strategies. Drawing on the emerging importance of information governance, this study examines the mechanisms through which it can facilitate competitive performance by aligning organizational capabilities. To test our proposed research model, we used survey data from 158 chief information officers and IT managers working in Norwegian firms. By means of partial least squares structural equation modeling (PLS-SEM), results show that information governance helps strengthen a firms' dynamic and operational capabilities, which in turn lead to competitive performance gains. The results are discussed in relation to their theoretical and practical implications.
How Does the Organizational Culture of Collaborative Networks Influence IT Governance Performance in a Large Organization?

In today's complex organizations, IT governance is an important managerial challenge. IT governance deals with decisions and responsibilities concerning IT. There are many factors influencing IT governance. One factor that has remained relatively unexplored by academic research is that of organizational culture. This research explores the influence of the organizational culture of collaborative networks on IT governance performance. A case study was conducted in a large complex company with several networks. The findings indicated that the networks desire different organizational culture types based on their priorities for IT governance outcomes. A clan organizational culture is desired when aiming for effective use of IT for asset utilization and cost effective use of IT. An adhocracy culture is desired when prioritizing the effective use of IT for business growth. Finally a combination of market and hierarchy organizational culture is desired when seeking effective use of IT for business flexibility and cost cutting.

\section{References}

[1] De Haes, S., Van Grembergen,W, Enterprise Governance of IT: Achieving Alignment and Value, Springer, 2015 